\title{
Comparison of reported dietary intakes between Caucasian and South Asian women and extent of under-reporting
}

\author{
H. Sanders, L. Tripkovic, L. Wilson, K. Hart and S. Lanham-New \\ Department of Nutritional Sciences, School of Biosciences and Medicine, Faculty of Health and Medical Sciences, \\ University of Surrey, Guildford, GU2 $7 X H$
}

The traditional dietary habits of differing ethnic groups vary greatly; concomitantly nutritional intakes are also likely to vary. Knowledge of differences in nutritional intakes between ethnic groups is important for understanding associated health risks. There is limited data on dietary intakes and patterns of South Asian populations. The aim of this study was to compare nutritional intakes of two ethnic groups; Caucasian (Cauc) and South Asian (SA).

Healthy Cauc and SA women, aged 20-64yrs, were recruited from the county of Surrey onto the D2-D3 study ${ }^{(1-3)}$ and a sub-set of these women were used in these analyses: $n 47$ Cauc and $n 47$ SA women. Anthropometrics and four-day food diaries were collected at baseline, as part of the D2-D3 study. Under-reporting of dietary intakes were determined by calculating energy intake (EI) to basal metabolic rate (BMR) ratio, and ratios below 1.35 were classified as under-reported ${ }^{(4)}$. Dietary and statistical analyses were carried out using DietPlan6(2013) and SPSS21(2013) respectively.

The Cauc women had significantly smaller waist circumferences than the SA women (Cauc:79.44 $\pm 11 \cdot 33 \mathrm{~cm}$ vs. SA:87.62 \pm 12.62 $\mathrm{cm}$; $p<0.001$ ), however BMI was not significantly different (Cauc: $24.60 \pm 3.75 \mathrm{~kg} / \mathrm{m}^{2}$ vs. SA: $25 \cdot 18 \pm 3.85 \mathrm{~kg} / \mathrm{m}^{2}$ ). As shown in Table 1, there were significantly lower daily intakes of micro-nutrients in the SA women compared to the Cauc women.

Table 1. Daily Dietary Intakes of South Asian (n47) and Caucasian (n47) women

\begin{tabular}{|c|c|c|c|c|c|c|c|c|c|}
\hline \multirow[b]{2}{*}{ Macro-nutrients } & \multicolumn{2}{|c|}{ South Asian } & \multicolumn{2}{|c|}{ Caucasian } & \multirow[b]{2}{*}{ Micro-nutrients } & \multicolumn{2}{|c|}{ South Asian } & \multicolumn{2}{|c|}{ Caucasian } \\
\hline & Mean & SD & Mean & SD & & Mean & SD & Mean & SD \\
\hline Energy KJ & $7432 \cdot 4$ & $2205 \cdot 6$ & $8269 \cdot 0$ & $1979 \cdot 7$ & Riboflavin $\mathrm{mg} * *$ & $1 \cdot 25$ & $0 \cdot 51$ & $1 \cdot 70$ & 0.60 \\
\hline Protein g & $67 \cdot 7$ & $24 \cdot 0$ & $74 \cdot 5$ & $15 \cdot 1$ & Thiamine $\mathrm{mg} * *$ & $1 \cdot 24$ & $0 \cdot 51$ & $1 \cdot 55$ & 0.43 \\
\hline Carbohydrate g & $203 \cdot 0$ & $69 \cdot 0$ & $223 \cdot 3$ & $62 \cdot 8$ & Vitamin B6 mg** & 1.60 & 0.72 & 1.98 & 0.60 \\
\hline Total Sugar g & $77 \cdot 5$ & $29 \cdot 7$ & $94 \cdot 5$ & $38 \cdot 7$ & Vitamin $\mathrm{B} 12 \mu \mathrm{g}$ & $4 \cdot 22$ & 3.41 & $4 \cdot 49$ & 2.63 \\
\hline Total Fat g & $75 \cdot 1$ & $28 \cdot 2$ & $77 \cdot 4$ & $25 \cdot 8$ & Vitamin $\mathrm{C} \mathrm{mg}$ & $91 \cdot 6$ & 57.9 & $102 \cdot 1$ & $57 \cdot 3$ \\
\hline Saturated Fat $g$ & $23 \cdot 58$ & $11 \cdot 56$ & 27.43 & $11 \cdot 18$ & Vitamin $\mathrm{D} \mu \mathrm{g}$ & $2 \cdot 07$ & 1.87 & 2.73 & $2 \cdot 51$ \\
\hline Trans-Fat g* & $1 \cdot 20$ & 0.72 & $1 \cdot 70$ & $0 \cdot 84$ & Folate $\mu \mathrm{g}^{*}$ & $220 \cdot 1$ & $111 \cdot 1$ & $265 \cdot 6$ & $89 \cdot 2$ \\
\hline Fibre $g^{* *}$ & $14 \cdot 8$ & $7 \cdot 9$ & $19 \cdot 7$ & $5 \cdot 7$ & Calcium mg** & 683.9 & $238 \cdot 7$ & $903 \cdot 2$ & $296 \cdot 0$ \\
\hline Alcohol g** & $0 \cdot 65$ & $2 \cdot 72$ & $10 \cdot 11$ & $11 \cdot 53$ & Sodium mg** & 2156 & 747 & 2849 & 737 \\
\hline & & & & & Potassium mg** & 2497 & 1009 & 3086 & 690 \\
\hline & & & & & Magnesium mg** & $237 \cdot 7$ & $91 \cdot 6$ & $292 \cdot 4$ & $64 \cdot 9$ \\
\hline
\end{tabular}

$* p<0.05 * * p<0.001$

Under-reporting of dietary intakes was present in $46.8 \%$ of Cauc women and $57.4 \%$ of SA women, however there was no significant difference between ethnic groups (EI:BMR ratios: Cauc:1.40 $\pm 0 \cdot 34$; SA: $1 \cdot 29 \pm 0 \cdot 38$ ).

These data suggest that although macro-nutrient intakes of these ethnic groups are similar, the foods from which these are sourced are likely to be different, thus contributing to the differences shown in micro-nutrient intakes. These differences in diets between the ethnic groups could be a contributing factor in the differing health and disease rates between ethnicities. The levels of under-reporting across both ethnic groups were high but nonetheless lower than those reported in our National surveys. Further analysis of the data is currently underway to investigate differences in intake when adjusted for total energy intake given

1. The D2-D3 Study: Ergocalciferol vs Cholecalciferol Food Fortification: Comparative Efficiency in raising 25OHD Status in Caucasian \& Asian women. Funded by the BBSRC DRINC (Grant No. BB/I006192/1).

2. Tripkovic et al. (2014) Proc Nutr Soc Submitted 2014.

3. Wilson et al. (2014) Proc Nutr Soc Submitted 2014

4. Goldberg et al. (1991) Eur J Clin Nutr 55, 569-81 